Les sources de l'Histoire des Mines : Nouveaux outils, Nouvelles approches

\title{
Les aveugles au travail. Des origines à l'émergence de nouveaux métiers à la fin du XIXe et au début du XXe siècle : sténographes, dactylographes, phono- dactylographes, téléphonistes
}

Blind people at work. From the origins to the emergence of new trades at the end of the XIX ${ }^{\text {th }}$ century and the beginning of the $X X^{\text {th }}$ century: stenographs, dactylographs, phono-dactylographs, telephonists

\section{Zina Weygand}

\section{OpenEdition}

\section{Journals}

Édition électronique

URL : http://journals.openedition.org/dht/668

DOI : $10.4000 /$ dht.668

ISSN : $1775-4194$

Éditeur :

Centre d'histoire des techniques et de l'environnement du Cnam (CDHTE-Cnam), Société des élèves du CDHTE-Cnam

\section{Édition imprimée}

Date de publication : 1 décembre 2008

Pagination : 89-100

ISBN : 978-2-95-30779-2-6

ISSN : $0417-8726$

Référence électronique

Zina Weygand, «Les aveugles au travail. Des origines à l'émergence de nouveaux métiers à la fin du XIXe et au début du XXe siècle : sténographes, dactylographes, phono-dactylographes,

téléphonistes », Documents pour l'histoire des techniques [En ligne], 16 | $2^{\mathrm{e}}$ semestre 2008, mis en ligne le 04 octobre 2010, consulté le 08 septembre 2020. URL : http://journals.openedition.org/dht/668 DOI : https://doi.org/10.4000/dht.668 


\title{
Les aveugles au travail.
}

\section{Des origines à l'émergence de nouveaux métiers à la fin du $\mathrm{XIX}^{\mathrm{e}}$ et au début du XXe siècle :} sténographes, dactylographes, phono-dactylographes, téléphonistes

\author{
Zina Weygand \\ CRTD-Cnam \\ CDHTE-Cnam
}

\begin{abstract}
RÉSUMÉ
Après avoir rappelé les grandes étapes de la théorisation puis de la mise en œuvre de l'intégration des aveugles dans le circuit des échanges socio-économiques par le biais de l'emploi, cet article - qui s'inscrit dans une réflexion sur la notion de « métier », proposée lors d'un séminaire transverse du Centre de Recherche sur le Travail et le Développement consacré en partie au métier de téléphoniste - expose les tentatives réalisées à la fin du XIXe et au début du XXe siècle pour permettre aux aveugles d'accéder à de nouveaux métiers, grâce aux innovations techniques qui transforment profondément alors l'univers des bureaux : machine à écrire, phonographe et téléphone.
\end{abstract}

Résumés et mots clés en anglais sont regroupés en fin de volume, accompagnés des mots clés français

Les aveugles au travail est le titre d'un ouvrage publié sous la direction de Philippe Chazal, directeur du Centre de formation et de rééducation professionnelle de l'Association Valentin Haüy'. Après diverses contributions de caractère général sur la déficience visuelle et l'emploi, qui constituent la première partie de cet ouvrage, la seconde partie rassemble une centaine de témoignages permettant de se faire une idée de l'éventail très large des professions susceptibles d'être exercées par des personnes aveugles à la fin du XX siècle - depuis les métiers de l'accueil et de la communication : interprète ; secrétaire; standardiste ; télé-conseiller - jusqu'au travail social : assistante sociale ; éducatrice spécialisée, en passant par différentes professions classées en plus d'une douzaine de rubriques. Si l'on trouve dans la rubrique "artisanat» quelques métiers qui, a priori, ne surprennent guère : brossier, chaisier, accordeur de pianos - mais encore faudrait-il s'interroger sur le bien-fondé de cette absence d'étonnement, comme si « depuis toujours», et

1 Philippe Chazal éd., Les aveugles au travail, Paris, éditions du Cherche Midi, 1999. encore aujourd'hui, ces métiers étaient des « métiers d'aveugles» - le lecteur non averti est plus étonné de découvrir un opérateur en salle de marché (qui depuis a d'ailleurs fait son chemin, puisqu'il est aujourd'hui Adjoint au Maire de Paris pour la Démocratie locale et la vie associative...) ; un câbleur, un opérateur sur machine dans l'industrie métallurgique; ou encore des magistrats; un médecin psychiatre, des ingénieurs, et même... un photographe, Michel Boisgontier, qui n'est d'ailleurs pas le seul aveugle à exercer ce métier'2. Le but de cet ouvrage est précisément de mieux informer les employeurs et les déficients visuels eux-mêmes sur le champ des activités professionnelles accessibles aux personnes aveugles, afin d' " ouvrir plus largement l'éventail des choix possibles $\|^{3}$.

\footnotetext{
2 Le plus célèbre est en effet le photographe d'origine slovène Evgen Bavcar, dont les travaux - qui envisagent les relations entre vision, cécité et invisibilité - ont fait l'objet de plusieurs expositions, en France et à l'étranger : « Mon propos est la réunion entre les mondes visibles et invisibles, la photographie me permettant de pervertir les modes de perception parmi ceux qui voient et ceux qui ne voient pas $»$. 3 Philippe Chazal, "Avant-propos ", dans Les aveugles au
} 


\section{Les aveugles au travail}

Si le lecteur peut être surpris par l'ampleur du champ des possibles révélé par cet ouvrage, on peut du moins espérer que plus personne aujourd'hui ne puisse douter de la pertinence de son tire : « Les aveugles au travail ». Pourtant, pendant des siècles, ces deux mots : " aveugles » et " travail » ont été antinomiques. Du reste, encore au XIX siècle, faute de pouvoir se fonder sur des critères optométriques (mesure de l'acuité et du champ visuel), on considérait comme complètement aveugle toute personne qui «n'y voyait pas à se conduire » et que son défaut de vision rendait « incapable d'aucun travail ». Le docteur Dumont, auteur d'un ouvrage sur les causes et les effets de la cécité, paru à Paris en 1856, définissait ainsi la cécité : « La cécité est la privation ou l'abolition de la vue. Cette infirmité existe toutes les fois qu'il est impossible de se livrer à aucun travail pour peu que ce travail réclame le concours - si faible qu'il soit - du sens de la vue $"{ }^{4}$. Aussi l'argument de l'inaptitude au travail revenait-il sans cesse dans les suppliques des postulants à une place aux Quinze-Vingts et sous la plume des médecins, des notables et des officiers municipaux qui soutenaient leurs demandes, puisque la définition de la cécité complète, exigée pour pouvoir être admis dans cet hospice - le seul réservé aux aveugles, depuis le Moyen Âge - était précisément basée sur des critères pratiques : entraves ambulatoires et professionnelles.

"Qui perd la vue perd tout», dit aussi un proverbe gascon, dont on peut retrouver l'écho, de nos jours, dans le slogan bien connu de la sécurité routière : «la vue, c'est la vie $»$ - et, de fait, pendant des siècles, la conviction largement partagée de l'inaptitude des aveugles au travail a incité les hommes et les femmes des classes laborieuses à considérer la cécité - qui les privait de toute ressource professionnelle-comme «la pire des infirmités». Au début du XIXe siècle, dans Le Visiteur du pauvre, le baron de Gérando résumait fort bien la situation des travailleurs pauvres confrontés à l'infirmité :

« Pour l'ouvrier qui vit de son travail, la santé est tout [...] ; si une infirmité vient à affecter l'un de ses organes [...], ceux dont sa profession exigeait un emploi habituel et exercé [...], d'un côté la source de ses modiques revenus est momenta-

travail, Paris, Le cherche midi éditeur, 1999, p. 16.

4 Georges Dumont, Recherches statistiques sur les causes et les effets de la cécité, Paris, Labé, 1856, pp. 8-9, cité par Zina Weygand, Les causes de la cécité et les soins oculaires en France au début du XIXe siècle (1800-1815), Paris, Ctnerhi , 1989, pp. 8-9. nément tarie ; de l'autre, de nouveaux besoins se font sentir, et il ne peut se suffire à lui-même. [...] Peut-être il demeure infirme pour le reste de ces jours, et alors il accroîtra les charges de [la] famille infortunée dont [...] il avait été le soutien. [...]. Malheureusement, un grand nombre de professions laborieuses exposent ceux qui les exercent à des maladies, à des accidents, à des infirmités prématurées $[\ldots] \gg{ }^{5}$.

Ce que ne voulaient pas voir Gérando et les observateurs sociaux de son temps, « prisonniers de leur profession de charité $\|^{6}$, c'étaient les réponses nouvelles (autres que l'assistance) qu'auraient pu apporter la société et les pouvoirs publics à la précarité des classes laborieuses... 7 . Pourtant, tout le travail des éducateurs et des philanthropes qui depuis Valentin Haüy, à la fin du XVIII e siècle, se sont efforcés d' " arracher " les aveugles des classes populaires à l'ignorance et à la mendicité par le biais de l'éducation et de l'apprentissage d'un métier, consistait à leur permettre d'accéder véritablement à l'emploi, d'une part en les persuadant eux-mêmes et en persuadant le public de leur aptitude au travail ; d'autre part, en permettant aux plus vulnérables d'entre eux d'exercer le métier qu'ils auront appris à l'école dans des ateliers gérés par les diverses sociétés de patronage créées au cours du XIXe siècle ; enfin, en essayant d'ajuster au marché du travail les formations professionnelles qui étaient proposées dans les institutions de jeunes aveugles.

"Les aveugles au travail ", une longue histoire donc, et un combat qui se poursuit de nos jours, avec encore bien des obstacles à contourner, dont le moin-

5 Joseph-Marie de Gérando, Le Visiteur du pauvre, $3^{e}$ édition, revue et augmentée, Paris, Jules Renouard, 1826, cité par Zina Weygand, "Les pauvres aveugles dans la société française (début du XIXe siècle) "), dans André Gueslin et Henri-Jacques Stiker éd., Handicaps, pauvreté et exclusion dans la France du XIXe siècle, Paris, Les éditions de l'Atelier, 2003, p. 58.

6 Louis Chevalier, Classes laborieuses et classes dangereuses à Paris pendant la première moitié du XIXe siècle, Paris, Hachette (Pluriel), 1984, p. 251.

7 Sur la philanthropie et les pauvres au XIX siècle, nous renvoyons à Philippe Sassier, Du bon usage des pauvres. Histoire d'un thème politique, $X V l^{e}-X X$ siècle, Paris, Fayard, 1990 ; Catherine Duprat, Usage et pratiques de la philanthropie. Pauvreté, action sociale et lien social à Paris au cours du premier dix-neuvième siècle, Paris, Comité d'histoire de la Sécurité sociale, 1996, 2 vol. ; André Gueslin, Gens pauvres, pauvres gens dans la France du XIXe siècle, Paris, Aubier, 1998 ; A. Gueslin, H.-J. Stiker éd., Handicaps, pauvreté et exclusion dans la France du XIXe siècle, op. cit. 
dre n'est pas le «préjugé » ou le « concept de cécité » mis en évidence par deux intellectuels aveugles du siècle dernier, Pierre Villey et Pierre Henri :

" Le concept de cécité se transmet et se comporte comme une force sociale, comme une de ces nombreuses représentations collectives qui dominent la connaissance et orientent les conduites. Il s'impose au voyant, le contraignant à penser d'une certaine manière en dépit de l'évidence et de l'expérience individuelle et commandant ses comportements à l'égard des aveugles. A ces derniers aussi il s'impose de l'extérieur, conditionnant leurs réactions et façonnant leurs mentalités de sorte que leur psychologie serait différente s'ils n'avaient pas à s'ajuster à ce concept ou à y résister $[\ldots] \|{ }^{8}$.

À notre connaissance, et pour ce qui concerne le monde occidental, l'idée que les aveugles sont susceptibles de s'intégrer par le travail au circuit des échanges socio-économiques, remonte au XVle siècle. En 1526, un humaniste espagnol vivant aux Pays-Bas, Juan-Luis Vives, fait paraître à Bruges un traité fondé sur la nouvelle morale religieuse - où le travail acquiert valeur de rédemption. Dans ce traité, De subventione pauperum - considéré comme l'œuvre maîtresse du programme humaniste de la réforme de l'assistance sociale - Vives entend imposer à tous la loi du travail salvateur, en intégrant dans la vie économique de la ville les groupes inactifs, afin de les rendre productifs. Il n'oublie pas les aveugles, bénéficiaires privilégiés de l'aumône et de fondations charitables durant les siècles précédents :

«On ne permettra pas même aux aveugles d'être ou d'aller oisifs; il y a beaucoup de choses en lesquelles ils peuvent s'exercer : les uns ont des dispositions pour les lettres, pourvu que quelqu'un lise pour eux. Qu'ils étudient [...]. D'autres sont aptes à la musique, qu'ils chantent et jouent des instruments à cordes et à vent; que d'autres fassent mouvoir des tours ou des rouages; que d'autres travaillent dans les pressoirs, aidant à manœuvrer les presses; que d'autres s'évertuent au soufflet

8 Pierre Henri, Les aveugles et la société, Paris, PUF, 1958, p. 32. II convient aussi de se reporter aux ouvrages de Pierre Villey : Le monde des aveugles. Essai de psychologie (1914), Paris, Librairie José Corti/GIAA, 1984 et L'aveugle dans le monde des voyants. Essai de sociologie, Paris, Librairie Flammarion, 1927, et à Zina Weygand, Vivre sans voir. Les aveugles dans la société française du Moyen Age au siècle de Lovis Braille, Grâne, Créaphis, 2003. dans les ateliers des forgerons ${ }^{9}$. On sait aussi que les aveugles fabriquent des boîtes, des paniers, des corbeilles et des cages et que les femmes aveugles filent et dévident. Bref, s'ils ne veulent chômer ni fuir le travail, ils trouveront aisément de quoi s'occuper; la paresse, la mollesse et non le défaut du corps est le seul motif qu'ils puissent alléguer pour ne rien faire ${ }^{10}$.

Dans ce texte, le premier à notre connaissance à donner une liste détaillée et argumentée des travaux intellectuels, artistiques et manuels accessibles aux aveugles, à une époque déterminée, Vives, partisan d'une désacralisation de la pauvreté et de l'aumône ayant pour conséquence la sécularisation de l'assistance, ne cite l'exemple des aveugles que pour mieux prouver le bien-fondé de sa théorie concernant la mise au travail des pauvres : si « même les aveugles », réputés « incapables d'aucun travail », peuvent exercer un certain nombre de travaux, manuels et autres, alors tout le monde peut travailler... Ce faisant, Vivès est le premier théoricien qui se refuse à traiter les aveugles différemment de « tout le genre humain »- auquel s'applique la loi « imposée par le Seigneur [...] comme peine et réparation de la faute originelle, à savoir : que chacun mange le pain acquis par sa sueur et son travail $॥ "$ ". Si le traité de Vives, qui servait le mouvement de réforme municipale de la bienfaisance, a connu en Europe une grande popularité, il fut pourtant sans effet en ce qui concerne la mise au travail des aveugles... ce qui dans l'immédiat fut plutôt à leur avantage, étant donné les conditions d'application des thèses mercantilistes aux mendiants valides dès cette époque. Les esprits, déjà partagés en ce qui concerne l'interdiction de la mendicité, étaient

9 Ce genre d'activités était sans doute encore proposé à la fin du XIXe siècle, aux aveugles n'ayant reçu aucune formation puisque Maurice de la Sizeranne écrivait en 1885 : " L'aveugle livré à lui-même, sans instruction, ne peut évidemment utiliser que la force mécanique de son bras : il tournera une meule, actionnera un soufflet de forge ou fera quelque chose d'analogue ; emplois dans lesquels il faut une force musculaire et une résistance à la fatigue peu communes pour arriver à un salaire rémunérateur ; en outre, l'être intelligent qui se livre à un tel travail ne tardera pas à s'abrutir ». M. de La Sizeranne, "Un mot sur l'utilisation du travail des aveugles ", communication au Congrès des unions de la Paix sociale et de la Société internationale d'Économie sociale (1885), Dix ans d'études et de propagande en faveur des aveugles, Tournon, Imprimerie de J. Parnin, 1890, p. 329. 10 Juan Luis Vives, De subventione pauperum, Bruges, 1526 ; Jean-Louis Vivès, De l'assistance aux pauvres, Bruxelles, Valéro et fils, 1943, pp. 206-207.

11 Id., p. 199. 


\section{Les aveugles au travail}

sans doute encore plus réticents à appliquer aux invalides - et en particulier aux aveugles - des mesures de travail forcé.

Nous pouvons cependant retenir des théories humanistes sur l'assistance qu'elles ont posé les premiers jalons d'une réflexion qui aboutira, à la fin du XVIII siècle, aux premières tentatives d'intégration sociale des aveugles pauvres par l'éducation et le travail. En 1783, la Société philanthropique, première fondation parisienne de bienfaisance libre, sans fins spirituelles ni direction ecclésiastique ${ }^{12}$, décide en effet « d'accorder un secours annuel à douze enfants de pauvres ouvriers, aveugles de naissance ou dès le bas âge ${ }^{13}{ }^{13}$, ceci afin de leur permettre de subsister jusqu'à l'âge de vingt et un ans - qui est alors la limite d'âge inférieure pour être admis à l'hospice des Quinze-Vingts. II n'est question ni d'éducation élémentaire ni d'apprentissage ; il s'agit au contraire de «tendre une main secourable " à des enfants auxquels leur infirmité « ravit l'espérance de pouvoir un jour subsister de [leur] travail $)^{14}$.

Cette décision et ces réflexions des membres de la Société philanthropique, publiées dans le Journal de Paris du 20 décembre 1783, leur attire les conseils d'Edme Régnier, «Mécanicien de S.A.S. Mgr le Duc de Chartres " ${ }^{15}$, sur la manière la plus efficace de venir en aide aux aveugles : "C'est beaucoup sans doute de contribuer à leur subsistance », reconnaît Régnier, « mais ne serait-ce pas faire plus encore pour eux de fonder leur subsistance sur leur propre travail ? ${ }^{16}$ » Aussi informe-t-il les Sociétaires « du grand nombre d'ouvrages différents dont ces malheureux sont capables» en leur en donnant « une liste fort abrégée et qu'on pourrait beaucoup augmenter en interrogeant des personnes instruites dans chaque partie $1{ }^{17}$.

12 La Maison philanthropique, bientôt dénommée « Société philanthropique ", a été fondée en 1780 par des maçons ayant à leur tête Savalette de Langes, fondateur de la loge des Amis Réunis, haut dignitaire du Grand Orient et organisateur de l'ordre des Philalèthes, ou " chercheurs de la Vérité ". 13 Journal de Paris, 1783/354 du 20 décembre 1783, « Bienfaisance ", " Aux auteurs du Journal [...] Un de vos Abonnés, Membre et Secrétaire actuel de la Société ॥, p. 1457

14 ld., p. 1456.

15 Louis-Philippe-Joseph, duc de Chartres, deviendra duc d'Orléans en 1785. Il est, à l'époque, Grand maître du Grand Orient de France.

16 Journal de Paris, 1784/111, 20 avril 1784, « Bienfaisance ॥, « Aux Auteurs du Journal, Semur en Auxois, 16 janvier 1784 [...]. Régnier, Mécanicien de S.A.S. Mgr le Duc de Chartres ", p. 234. Sur Edme Régnier, voir ci-dessous. 17 Ibid.
Cette liste est, à notre connaissance, la seconde après celle de Vives, qui répertorie les activités susceptibles d'être exercées par les aveugles à une époque donnée :

« Raper du tabac ; piler du ciment ; moudre ou pulvériser dans des mortiers ; tourner une meule, une rove pour faire aller une pompe, une petite forge ; souffler l'orgue ; polir le marbre, l'acier, le bois ; faire des chaînes de fil de fer avec une pince ; empailler des chaises ; faire des paillassons ... ; éplucher le crin ; carder la laine, le coton ; faire des mèches, de la tresse, des lacets, des filets pour prendre oiseaux et poissons ; tricoter des jarretières ... ; faire des boutons de poil de chèvres ... ; travailler le chanvre ; filer ; faire des cordes ... ; sans parler de la musique, soit vocale, soit instrumentale ; langues, grammaire ; lire, écrire, faire de la géométrie et même de l'optique, témoin Saunderson, en employant des caractères, des figures mobiles et en relief ; enfin, toutes les sciences abstraites $1{ }^{18}$.

Ce texte est édifiant à plus d'un titre : d'une part, il témoigne des connaissances dont était susceptible un citoyen éclairé de la fin du XVIII ${ }^{e}$ siècle ${ }^{19}$ sur les capacités des aveugles à exercer un certain nombre d'activités très diverses; d'autre part, il nous permet de constater que l'éventail des métiers manuels susceptibles d'être exercés par des aveugles s'était élargi depuis Vives; mais nous pouvons constater également combien, dans ce domaine, les tâches proposées aux aveugles étaient peu qualifiées et, de ce fait, peu lucratives. Ce problème restera la préoccupation majeure des éducateurs et des hommes d'œuvres dévoués à la cause des aveugles, pour ce qui concerne l'emploi des ouvriers aveugles, durant tout le XIXe et une partie du XXe siècle.

\section{$18 \mathrm{lbid}$.}

19 Edme Régnier, né en 1751 à Semur-en-Auxois, fils et petit-fils de serrurier, inventeur de diverses armes et serrures récompensées par la société de l'abbé Baudeau, venait de recevoir le brevet de « mécanicien de la province de Bourgogne et de Monseigneur le Duc de Chartres " pour l'invention d'une machine à filer et à câbler les cordes de fer. Par ailleurs, il avait offert à Louis XVI, l'année précédente, un méridien à canon, sonnerie et musique. Sur Régnier, on peut se reporter à Liliane Hilaire-Pérez, L'invention technique au siècle des Lumières, Paris, Albin Michel, 2000, et à Patrice Bret, L'État, l'armée, la science. L'invention de la recherche publique en France (1763-1830), Rennes, PUR, 2002, qui l'évoquent à plusieurs reprises, et à Alain Mercier, Le portefeuille de Vaucanson. Chefs d'œuvre du dessin technique, Paris, Musée national des techniques, 1991, pp. 42-43. 
Quoi qu'il en soit, les suggestions de Régnier - qui rencontraient le souci d'utilité sociale dont se réclamait toute démarche vraiment philanthropique auront plus d'impact que les théories de Vives en leur temps ; ainsi, la première répartition du secours annuel accordé aux enfants aveugles par la Société philanthropique fera-t-elle une part importante à l'achat « des instruments propres à leur enseigner quelque métier ». La rencontre des Sociétaires et de Valentin Haüy, interprète du Roi et de l'Amirauté, membre du Bureau académique d'écriture depuis 1781, et porteur d'un projet d' « institution » des aveugles, fera le reste. Mais ce n'est pas ici le lieu d'en parler, sinon pour rappeler les divers travaux manuels auxquels le fondateur de I'Institution des enfants aveugles, créée à Paris en 1785 avec l'aide de la Société philanthropique, a appliqué ses élèves : en premier lieu, l'imprimerie, apprise sous la direction de Clousier, imprimeur du roi et membre de la Société philanthropique, puis « diverses occupations relatives aux Arts et aux Métiers »:

"On les appliqua avec succès à la Filature [" à

l'aide d'une machine fort ingénieuse de l'invention du S. Hildebrand, Mécanicien »]. Du fil de leur fabrique nous réussîmes à leur faire retordre de la ficelle ; et de cette ficelle, nous leur fîmes tramer de la sangle. Les ouvrages au boisseau, le filet, le tricot, la couture, la reliure des livres, tout fut tenté à notre satisfaction ; et nous manquâmes plutôt d'artisans que de travaux : tant il est d'espèces d'occupations manuelles que l'on peut confier aux aveugles $1{ }^{20}$.

Malheureusement - à quelques variantes près pour ce qui concerne leur nature : abandon des ouvrages au boisseau, de la couture, de la reliure ; introduction de nouveaux métiers : tisseranderie, vannerie, rempaillage et cannage de sièges, fabrication de tapis de paille, brosserie ; fabrication de bourses et d'objets en perles pour les filles, etc. - une constante se dégage, concernant les travaux manuels pratiqués par les aveugles au cours du XIX et au début du $X X$ siècle : leur faible rentabilité. En ce domaine, les ouvriers aveugles se heurteront en effet à trois grandes difficultés : le problème de l'achat en gros des matières premières, celui de la rapidité de production lles ouvriers aveugles ont du mal à lutter de vitesse avec les clairvoyants), enfin celui de l'écoulement des marchandises. En un mot, il restera difficile de trouver des métiers manuels suffisamment lucratifs pour permettre

20 Valentin Haüy, Essai sur l'éducation des aveugles, 1786 - Editions des Archives Contemporaines, 1985, (collection Bibliothèque du Cnam), p. 93. aux ouvriers aveugles de se suffire à eux-mêmes - et il faudra le plus souvent les regrouper dans des établissements « ayant le double caractère d'atelier de production et de magasin de vente $\|{ }^{21}$.

À cet égard, un seul métier, intermédiaire entre les carrières artistiques et les professions manuelles, fait exception : celui d'accordeur-facteur de pianos, " officiellement » ouvert aux aveugles par la création d'une classe d'accord à l'Institution nationale de Paris en 1836, à la suite du succès professionnel rencontré dans ce domaine par Claude Montal, élève puis répétiteur à l'Institution royale des jeunes aveugles de 1817 à 1830 22. Ce métier restera longtemps, avec la profession d'organiste, la seule carrière vraiment lucrative pour les aveugles.

Nous allons précisément aborder maintenant les tentatives réalisées à la fin du XIXe et au début du $X X{ }^{e}$ siècle pour ouvrir de nouveaux métiers - si possible plus lucratifs - aux aveugles insuffisamment doués pour la musique pour en faire leur profession, - y compris comme accordeurs de pianos, d'un niveau scolaire trop " moyen " pour devenir enseignants, mais suffisamment instruits pour être préposés « à des travaux présentant un caractère d'ordre plutôt intellectuel que matériel ${ }^{23}$. En un mot pour devenir des " employés", cet " autre de l'ouvrier ", pour reprendre l'expression de Delphine Gardey dans son ouvrage sur I'histoire des employés de bureau ${ }^{24}$. A ces tentatives ont contribué notamment, en France - mais ils ne furent pas les seuls - les typhlophiles regroupés au sein de l'Association Valentin Haüy, fondée entre 1883 et 1889 par un aveugle, Maurice de La Sizeranne (18571924), qui en fut le secrétaire général de 1889 à 1924 ,

21 M. de La Sizeranne, "Un mot sur l'utilisation du travail des aveugles», op. cit., p. 332.

22 Sorti de l'Institution en 1830 pour exercer la profession d'accordeur puis celle de facteur, Claude Montal crée sa propre entreprise et réussit tellement bien, après des débuts difficiles, qu'en 1853 il pourra s'honorer du titre de " fournisseur breveté de LL.MM. l'Empereur et l'Impératrice des Français " (Pierre-Armand Dufau, Paul-Emile Bienaimé et M. Tahan, Claude Montal, facteur de pianos (aveugle) : sa vie et ses travaux, Paris, Firmin Didot, 1857, p. 15, cité par $Z$. Weygand, Vivre sans voir, op. cit., p. 325).

23 A. Artaud, La question de l'employé en France, étude sociale et professionnelle, Librairie Georges Roustan, 1909, p. 3, citation extraite du Traité de législation industrielle de Paul Pic, dans Delphine Gardey, La dactylographe et l'expéditionnaire. Histoire des employés de bureau, 1890-1930, Paris, Belin, 2001, p. 20.

24 Delphine Gardey, op.cit., p. 20. 


\section{Les aveugles au travail}

et auquel succéda un autre grand leader aveugle, Pierre Villey (1879-1933), normalien, professeur de lettres à la Faculté de Caen, éditeur des Essais de Michel de Montaigne et auteur de trois grands livres sur les aveugles : Le monde des aveugles (1914), La pédagogie des aveugles (1922) et L'aveugle dans le monde des voyants (1927).

L'émergence de ces nouveaux métiers est liée à trois inventions techniques majeures de la fin du XIXe siècle dans les domaines de la production de l'écriture, de la reproduction de la parole et de la communication à distance : le type-writer de Christopher Sholes et Carlos Glidden, manufacturé par Remington en 1874 ; le phonographe ${ }^{25}$ et le téléphone ${ }^{26}$, et à leur entrée dans «l'univers des bureaux, un monde [alors] en pleine mutation $»{ }^{27}$, comme le souligne Delphine Gardey : « Les activités administratives s'affirment, les entreprises industrielles et les commerces s'organisent à une plus large échelle, les emplois augmentent considérablement en même temps qu'ils se diversifient et se féminisent $"{ }^{28}$. Pourquoi les " outils matériels nouveaux " ${ }^{29}$ - machines à écrire, phonographe commercial, téléphone, qui accompagnent cette "révolution administrative " - ne permettraient-ils pas aux aveugles d'accéder à des emplois et à un univers professionnel qui leur étaient jusque là fermés ? C'est la question que se posent manifestement les auteurs de plusieurs articles parus à la fin du XIXe et au tout début du XXe siècle dans la revue Le Valentin Haüy, fondée en 1883 par Maurice de La Sizeranne, à l'attention de « toutes les personnes qui, à un titre quelconque, s'occupent de l'éducation, de l'enseignement, de l'apprentissage ou de l'emploi des aveugles $1{ }^{30}$.

Ainsi les numéros d'avril 1893 et mai 1894 de la revue rapportent-ils les propos tenus par un certain M. Bournizien, avocat à la cour d'appel de Paris et président de l'association des sténographes français. Lors de deux réunions de la Conférence Valentin Haüy, le 23 mars 1893 et le 19 avril 1894, M. Bournizien a prétendu que des aveugles intelligents, ayant reçu

25 Inventé en 1877 par Edison qui, en association avec Bell, réalisa en 1888 le phonographe commercial.

26 Inventé par Graham Bell en 1876

27 Delphine Gardey, « Mécaniser l'écriture et photographier la parole. Utopies, monde du bureau et histoires de genre et de techniques 1), Annales HSS, mai-juin 1999, n³, pp. 587-614. 28 Ibid.

29 Ibid.

30 Maurice de La Sizeranne, "Aux amis des aveugles 》, Le Valentin Haüy. Revue française des questions relatives aux aveugles, $n^{\circ} 1$, janvier 1883, 1 ière année, p. 1. une instruction assez étendue, pourraient apprendre à manier avec grande dextérité la machine à écrire (Remington, Hammond, Calligraph) afin de gagner honorablement leur vie (10 à 12 francs par jour), comme "typoscribes », c'est-à-dire en écrivant à la machine sous la dictée des sténographes, les discours et les débats parlementaires ou judiciaires.

À la réunion du 23 mars 1893, M. Bournizien a d'ailleurs émis l'idée « que les aveugles pourraient être [également] employés comme sténographes commerciaux $1{ }^{31}$. Cette idée fera son chemin et suscitera l'invention de plusieurs machines à sténographier pour les aveugles. Par exemple, la sténophile Bivort, présentée dans des extraits de la revue mensuelle La sténomécanographie, de janvier à mai 1914, rassemblés en une plaquette intitulée « Nouvelles carrières pour Aveugles " : "La machine à sténographier "Sténophile-Bivort ॥ permet [...] à tout aveugle de devenir comme les vrais voyants [sic], en très peu de temps, un habile sténographe, sûr et rapide ${ }^{32}$.

Pour preuve de l'efficacité de cette invention, l'article donne l'exemple d'un aveugle de 28 ans, M. Lejeune, qui occupe un emploi de téléphoniste et « note avec sa machine les principales communications ; il prend de même la dictée d'un courrier important $॥{ }^{33}$. Devenu « un virtuose de la Sténophile [...] il peut lutter comme rapidité et sûreté avec les meilleurs sténographes voyants. Chose plus remarquable encore M. Lejeune a formé, seul, et par son initiative, plusieurs élèves sténophilistes qui ont pu obtenir des emplois bien rémunérés $1{ }^{34}$. À propos des performances de cet aveugle, l'auteur de l'article cite plusieurs concours où il a été distingué, comme dactylographe et comme sténographe. En fin de plaquette, une note bas de page nous informe du fait que «M. Lejeune vient d'être désigné comme professeur de sténographie et de dactylographie à la société : Les Amis des Soldats aveugles, installée dans la maison de convalescence de Revilly (mai 1915) $1{ }^{35}$.

Quelques années auparavant, était paru, dans le numéro de septembre 1899 du Valentin Haüy, un arti-

31 Le Valentin Haüy, n 4, avril 1893, p. 44. Compte rendu de la Conférence Valentin Haüy du 23 mars 1893.

32 « Nouvelles Carrières pour Aveugles», Extraits de la revue mensuelle La sténo-mécanographie, janvier à mai 1914, p.1. 33 lbid.., p.2

34 lbid.

35 Id., p. 3, note 1. Sur la carrière de Germain Lejeune, on peut lire la notice insérée dans l'ouvrage de Delphine Jaunasse sur l'École Braille, A la lumière des temps. Histoire et perspectives d'un établissement fondé pour les aveugles (1883-2000), Saint-Mandé, Institut Le Val Mandé, 1999, p. 46. 
cle intitulé : « La dactylographie ». Après avoir déploré la force du « préjugé » qui, en France, faisait obstacle à l'embauche de dactylographes aveugles, alors que « la machine à écrire, adoptée maintenant par tant de clairvoyants, a été inventée tout d'abord pour les aveugles $)^{36}$ - l'auteur citait l'exemple de l'Angleterre, où M. Stainsby, directeur de l'Institution d'aveugles de Birmingham, avait créé un Bureau de dactylographes aveugles. Dans cet établissement, dirigé par une clairvoyante et équipé « de toutes les commodités modernes nécessaires pour le travail, telles que nouveaux types de phonographes, de téléphones et une variété choisie des types les plus nouveaux des machines Remington $1{ }^{37}$, les aveugles exécutaient tous les travaux susceptibles de se faire à la machine à écrire. Pour prendre rapidement sous la dictée, ils employaient une sténographie braille ; ils étaient également capables de transcrire directement à la machine des textes enregistrés sur un phonographe.

Pour ce qui concerne l'usage couplé du phonographe et de la machine à écrire par les aveugles, deux ans plus tard, en août 1901, le Valentin Haüy proposait à ses lecteurs un article de Constant Aussel sur "Le phonographe et la machine à écrire », dans lequel l'auteur citait en exemple M. Gariel, directeur du journal Le Petit Méridional et membre de l'Association Valentin Haüy de Montpellier, qui « a bien voulu consentir à accepter, dans les bureaux de son administration, un aveugle affecté à la correspondance, transcriptions d'ordres, instructions, observations au

36 H. de La Brière, "La dactylographie ", Le Valentin Haüy, n9, septembre 1899, p. 75. A ce propos, l'auteur fait un développement sur la machine à écrire de Pierre-François Foucault. Sur les mécaniques à écrire inventées par Foucault, nous renvoyons à notre article : « Un clavier pour les aveugles ou le Destin d'un inventeur : Pierre François Victor Foucault (1797-1871) 1), Voir barré, n²3, décembre 2001, pp. 30-41. Pour sa part, Delphine Gardey, dans l'article cité plus haut, précise également que les premières machines à écrire avaient d'autres finalités que commerciales : réaliser chez soi des caractères similaires à ceux d'une imprimerie ; répondre aux besoins spécifiques des aveugles - outre les machines de Foucault, elle fait allusion au Typographe de l'américain Hughes (1850), utilisé dès 1851 dans des institutions d'aveugles. Enfin, elle rappelle que « le Typewriter Remington [s'inscrivait] globalement dans la lignée des machines précédentes et [qu'il était] proposé aux sténographes des tribunaux [...], aux hommes de loi, aux éditeurs, aux auteurs, aux prêtres, aux copistes, aux aveugles, aux écoliers, aux télégraphistes et aux hommes d'affaires "; D. Gardey, "Mécaniser l'écriture et photographier la parole ॥, op.. cit., pp. 608-609. 37 H. de La Brière, « La dactylographie », op.cit., p. 75. personnel des divers services du journal $॥{ }^{38}$. L'auteur de cet article précisait en outre que « les différents travaux précités [étaient] enregistrés sur phonographe et transcrits ensuite à la machine à écrire ${ }^{39}$; il décrivait ensuite le fonctionnement de «l'installation téléphonographique qui [existait] au Petit Méridional » : grâce à cette installation,

«les informations téléphoniques sont transmises par le correspondant de Paris ou de la province et répétées au fur et à mesure dans un entonnoir de verre muni d'un double tube coudé, communiquant l'un à la plaque microphonique et l'autre au phonographe. De cette façon, en même temps que la personne qui transmet les communications se rend compte qu'on a bien entendu ce qu'elle vient de dicter, se fait l'inscription au phonographe du texte répété [...]. II ne reste plus alors qu'à transcrire à la machine à écrire ce qui a été impressionné sur le cylindre, opérations qui se font toutes très aisément et surtout très rapidement. Dans son numéro du 14 mars 1900 - précise Constant Aussel - le Petit Méridional a consacré un très long article à ce nouveau métier, sous la rubrique "Les aveugles téléphonographistes ${ }^{40}$.

Cet article sera reproduit en 1903 dans un nouvel article du Valentin Haüy intitulé : « La dactylo-téléphonographie et les aveugles $\gg{ }^{41}$.

Ainsi donc, peu à peu, l'univers des bureaux, soumis à de nouvelles exigences - notamment de rapidité et de rendement - répondant à de nouvelles normes de production des écritures administratives, s'ouvre-t-il aux aveugles, et ce grâce à l'apparition des matériels nouveaux permettant la mécanisation de l'écriture, l'enregistrement de la parole et sa transmission à distance.

Les expériences mises en valeur par les articles du Valentin Haüy restent toutefois ponctuelles et les auteurs de ces articles voudraient convaincre les industriels et les hommes d'affaires français d'être moins frileux vis-à-vis de l'emploi des aveugles dans les services administratifs de leurs entreprises.

Dans les pays anglo-saxons, Royaume-Uni, ÉtatsUnis, il semblerait en effet que l'on ait été plus hardi à cet égard. Outre l'article du Valentin Haüy de septembre 1899 citant la création d'un bureau de dactylographes aveugles à Birmingham, nous avons trouvé à la Bibliothèque Valentin Haüy un article du Family herald

38 Constant Aussel, « Le phonographe et la machine à écrire "), Le Valentin Haüy, 1901/8, p. 88.

39 lbid.

40 ld., pp. 88-89

41 Le Valentin Haüy, 1903/8, p. 73-77. 


\section{Les aveugles au travail}

weekly star de Montréal, daté du 27 novembre 1907 , concernant l'emploi de sept téléphonistes aveugles à New York City, dont l'une était en poste depuis environ deux ans, et les six autres depuis moins d'un an. C'est du reste le succès de la première qui a incité la New York Association for the Blind à convaincre une compagnie téléphonique de former les six autres (quatre hommes et deux femmes). L'une d'elles n'étant pas occupée à plein temps par le standard exécute également des travaux de sténo-dactylographie, et utilise pour cela une machine à sténographier anglaise de l'invention de Henry Stainsby... le créateur du bureau de dactylographes aveugles de Birmingham - dont le Family Herald souligne qu'il a été également le premier à placer un aveugle comme téléphoniste, en Angleterre.

En France, si l'on en croit ce qu'écrit Pierre Villey en 1922, dans La pédagogie des aveugles, c'est la guerre de 1914-1918 qui a transformé l'état d'esprit des employeurs vis-à-vis des aveugles :

« [...] la guerre nous a subitement placés dans des conditions d'expérimentation singulièrement propices. Un très grand nombre d'aveugles se sont présentés à la fois dans des écoles de rééducation afin d'y être pourvus d'un métier. [...] Pardessus tout, l'état d'esprit des employeurs était transformé ; imaginez que, en pleine paix d'avant 1914, vous alliez proposer à un chef d'usine ou à un contremaître de recevoir un aveugle parmi leurs ouvriers. Vous devinez sans peine l'accueil que vous auriez reçu : un accueil poli, peut-être aimable, mais parfaitement décourageant La guerre a changé cela; elle a ouvert toutes les portes devant les militaires aveugles, et fait un devoir à tout homme de conscience de ne les évincer qu'après les avoir écoutés.

Aussi, des métiers dont nous commencions seulement en France à faire l'essai - la tonnellerie dont l'Association Valentin Haüy avait facilité I'apprentissage à plusieurs sujets, la cordonnerie - ont pris soudain un essor inattendu. Les téléphonistes aveugles se sont multipliés. Des métiers nouveaux aussi ont été tentés qui ont paru donner des résultats favorables [...] $11{ }^{42}$.

Villey s'interroge malgré tout : « Que pourrons-nous conserver demain des positions acquises?")

Pour les métiers qui nous intéressent, si l'on en croit les chiffres de l'Union des Aveugles de guerre donnant la répartition par métiers des soldats aveugles sortant

42 Pierre Villey, La pédagogie des aveugles, Paris, Librairie Félix Alcan, 1922, nouvelle édition, s. d., pp. 190-191. de rééducation - ce qui ne veut nullement dire que c'est la profession qui sera effectivement exercée - on trouve seulement 30 téléphonistes et 20 dactylographes sur un total de 1742 soldats rééduqués (la guerre aurait fait 3000 aveugles) ${ }^{43}$. C'est encore peu...

Quoi qu'il en soit, c'est en 1917 que l'Association Valentin Haüy met en place la première formation pour standardistes aveugles. On peut d'ailleurs lire dans Le Valentin Haüy de Juillet 1917 un court encart sur «Les aveugles téléphonistes »:

«Avant la guerre on étudiait déjà cette utilisation.

Aujourd'hui, c'est un fait reconnu qu'un aveugle peut remplir le poste de téléphoniste dans toute administration, magasin, usine etc., possédant un réseau privé. C'est donc un devoir de réserver cet emploi aux soldats aveugles auxquels désormais tant de carrières sont fermées.

L'Association Valentin Haüy demande au public de bien vouloir lui apporter son concours pour les encourager dans cette voie et pour l'aider au placement de plusieurs d'entre eux. Elle peut fournir divers exemples, notamment à Toulouse, Marseille, aux usines de Saut-du-Tarn, et au siège même de l'œuvre, 9, rue Duroc, où un Chasseur à pied, blessé en Champagne, opère, avec une parfaite aisance sur un standard de 45 numéros.

[...] 1$)^{44}$.

C'est cet aveugle de guerre qui est bientôt chargé de la formation de ses confrères. Dès lors, l'œuvre n'a de cesse de faire de la propagande pour l'embauche des téléphonistes aveugles.

Cependant, il faut attendre l'apparition puis l'évoIution d'une législation en faveur de l'emploi des personnes handicapées, depuis la loi du 26 avril 1924 sur l'emploi obligatoire des mutilés de la guerre et des accidentés du travail dans le commerce et l'industrie jusqu'à la loi du 23 novembre 1957 sur l'obligation d'emploi des personnes handicapées - que suivra, en 1960, la création de l'Amicale des standardistes aveugles de France, dont tous les efforts porteront sur la formation professionnelle, le placement et l'aménagement technique des postes de travail - pour que l'embauche des standardistes aveugles atteigne dans notre pays des chiffres vraiment significatifs ${ }^{45}$.

43 Jean-François Montès, 1915-1939, (re)travailler ou le retour du mutilé : une histoire de l'entre-deux-guerres. Rapport de recherche effectué pour l'Office national des anciens combattants et victimes de guerre, Hôtel national des Invalides, décembre 1991, p. 84.

44 "Les aveugles téléphonistes », Le Valentin Haüy, juillet $1917, n^{\circ} 2$, p. 12

45 D'après un entretien avec M. Roger Toupet, vice-président 


\section{Zina Weygand}

Cette évolution est une autre histoire, qui se poursuit aujourd'hui avec la mise en œuvre d'un nouvel appareil législatif ${ }^{46}$, l'apparition de nouvelles techniques notamment informatiques - et le développement des métiers de l'accueil et de la communication ${ }^{47}$.

Cet article issu d'une communication présentée lors d'une journée d'étude proposée par le Centre de Recherche sur le Travail et le Développement du Cnam sur la question du « métier », a tenté de retracer l'évolution des représentations, des théories et des pratiques concernant l'intégration des aveugles au circuit des échanges socio-économiques par le biais de l'emploi - depuis les théories humanistes de l'assistance jusqu'aux actions entreprises par les associations d'aveugles à la fin du XIXe et au cours du XXe siècle pour permettre au plus grand nombre d'accéder à la dignité par le travail - en passant par les réalisations des philanthropes du XVIII et du XIXe siècle en faveur de la formation professionnelle et de la mise au travail des aveugles pauvres.

Or cette évolution, qui a abouti, à la fin du XIXe et au début $d u X X$ siècle à l'émergence de nouveaux débouchés professionnels pour les aveugles - notamment dans le monde des bureaux - a été possible grâce aux innovations techniques qui ont accompagné les réflexions et les efforts des philanthropes en faveur de l'emploi des aveugles - et ce dès la fon-

de l'Amicale des Standardistes Aveugles chargé du placement de 1960 à 1986, le nombre de personnes aveugles placées était passé, entre ces deux dates, de trois ou quatre par an, pour toute la France, à une centaine. Entretien avec M. Roger Toupet, vice-président fondateur de l'Amicale des Standardistes Aveugles de France, (ASAF, devenue ASATAF : Amicale des Standardistes et Agents Techniques Aveugles de France), 4 février 2008.

46 Loi n 2005-102 du 11 février 2005 pour l'égalité des droits et des chances, la participation et la citoyenneté des personnes handicapées.

47 Entretien avec $M^{\text {me }}$ Joëlle Lecoq, présidente actuelle de I'Amicale des Standardistes et Agents Techniques Aveugles de France, 24 janvier 2008. dation de l'Institut des enfants aveugles par Valentin Haüy en 1785.

II serait intéressant d'étudier plus en détail la part prise par les aveugles eux-mêmes, dès le premier tiers du XIXe siècle et au début du XXe siècle, aux innovations qui ont permis au plus grand nombre d'accéder à l'éducation, à la communication et à l'emploi. Ainsi, bien sûr, de l'appropriation puis de la réinvention par le jeune Louis Braille d'un système d'écriture ponctuée conçu par un philanthrope clairvoyant, Charles Barbier de La Serre ; ainsi des mécaniques à écrire de Foucault ; ainsi, de l'adaptation de la sténophile Bivort devenue, en 1917, machine à sténographier Bivort-Villey-Lejeune, grâce aux échanges entre l'inventeur, un clairvoyant, et deux aveugles, Pierre Villey et Germain Lejeune - et de bien d'autres nouveautés techniques mises au point à cette époque par des aveugles de talent.

Ces inventions réalisées par des aveugles pour les aveugles sont à notre avis l'une des manifestations de l'émergence d'une prise de conscience identitaire, née au sein de la communauté des aveugles instruits, durant le premier tiers du XIXe siècle ; elle aboutira à la fin du siècle à la création de la première association fondée par un aveugle pour les aveugles : l'Association Valentin Haüy, fondée par Maurice de La Sizeranne entre 1883 et 1889, qui précisément soutiendra les innovations techniques en faveur des aveugles et les fera connaître aux personnes intéressées par des articles publiés dans ses périodiques et par une exposition permanente présentée au public dans un petit musée créé spécialement à cet effet en 1886.

Les périodiques spécialisés dans le domaine de la pédagogie des aveugles et de la propagande en leur faveur, créés au cours du XIXe siècle; les archives et les objets conservés au musée Valentin Haüy, mais également les archives des instances de légitimation de l'invention; les publications des expositions universelles; les actes des colloques spécialisés qui se sont réunis à partir de la seconde moitié du XIXe siècle, constituent les sources sur lesquelles pourrait s'appuyer ce type de recherche. 


\section{BibLIOGRAPHIE}

Association Valentin Haüy, Plaquette de présentation du Centre de Formation et de Rééducation Professionnelle. «Présentation générale du Centre. Historique », s.d.

Chazal, Philippe (dir.) (1999), Les Aveugles au travail, Paris, Le Cherche Midi, $307 \mathrm{p}$.

Clot, Yves (dir.), Prot, Bernard, Reille-Baudrin, Emmanuelle (2007), Entre le référentiel et l'activité : le problème de la prospective du métier. Une étude avec des employés administratifs. Rapport d'étude au ministère de l'Education nationale, Programme d'étude 2004-2005, thème 2 : « L'évolution des professions, des métiers et des activités professionnelles $»$, CPC documents, année 2007, n¹, 97 p.

Dolza, Luisa, Hilaire-Pérez, Liliane, Weygand, Zina. (2006), " Les institutions d'assistance auX XVIII" et XIXe siècles à Paris et à Turin. Des ateliers entre rentabilité, philanthropie et expérimentation », in Le Travail avant la révolution industrielle. Actes du $127^{\mathrm{e}}$ congrès des sociétés historiques et scientifiques (Nancy, 2002), pp. 325-343 (version électronique).

« Formation et emploi des aveugles en GrandeBretagne » in Revue internationale du travail, vol. LXIVn²-3, Août-Septembre 1951, pp. 251-259.

Gandon, Annie (1979), Pédagogie des handicapés. La Formation professionnelle des handicapés visuels, Mémoire [la discipline n'est pas indiquée], 102 p.

Gardey, Delphine (2001), La Dactylographe et l'expéditionnaire. Histoire des employés de bureau 18901930, Paris, Belin, 336 p.

Gardey, Delphine (1999), " Mécaniser l'écriture et photographier la parole. Utopies, monde du bureau et histoires de genre et de techniques 1 , in Annales HSS, 1999/3, pp. 587-614.

Henri, Pierre (1958), Les Aveugles et la société. Contribution à la psychologie sociale de la cécité, Paris, P.U.F., 465 p.

Hilaire-Pérez, Liliane (2000), L'invention technique au siècle des Lumières, Paris, Albin Michel, (L'évolution de l'humanité), 443 p. (Préface de Daniel Roche).

Jaunasse, Delphine (2000), A la lumière des temps. Histoire et perspectives d'un établissement fondé pour les aveugles (1883-2000), Institut le Val Mandé, 165 p.

"La poste et les non-voyants » in Le Valentin Haüy, 1993/29, p. 16.

L'emploi des aveugles dans les administrations publiques, Actes des Journées d'études des 23, 24 et 25 juin 1955 tenues sous la présidence de M. Paul Haag,
Préfet de la Seine et organisées par l'Institut Technique des Administrations Publiques, $89 \mathrm{p}$.

« Les personnes ayant un handicap visuel. Les apports de l'enquête Handicaps-Incapacités-Dépendance ॥, Drees, Études et résultats, n 416, juillet 2005, 12 p.

Lutten L.-P. (1962), « Des aveugles à la croisée de mille invisibles chemins ॥ in L'Alsace illustrée de Mulhouse, Reportage de L.-P. Lutten [sur des aveugles standardistes], pp. 5-7.

«MACIF, Provence-Méditerranée, Le nouveau centre de renseignements téléphoniques de Marseille $»$, Le Valentin Haüy, 1991/23, pp. 30-31.

Montès, Jean-François (1991), 1915-1939, (re)travailler ou le retour du mutilé : une histoire de l'entredeux guerres. Rapport de recherche effectué pour I'Office National des Anciens Combattants et Victimes de guerre, Hôtel National des Invalides, 1991, 438 p.

Montès, Jean-François (1990), "La rééducation professionnelle et l'emploi des aveugles entre 1880 et 1945 ॥, in Le Valentin Haüy, 1990/19, pp. 18-21.

Montès, Jean-François (1990), "Les aveugles de guerre. Le problème de leur rééducation et de leur emploi ॥, in Le Valentin Haüy, 1990/20, pp. 6-9.

Pacaud, Suzanne (1949) « Recherches sur le travail des téléphonistes. Étude psychologique d'un métier »", in Le travail humain, 1949, XII, 1-2, pp. 46-65.

«Repères sur les personnes atteintes de déficience visuelle - Vol.Il : Formation professionnelle ; Travail et emploi ; Accessibilité ॥, Dossier professionnel documentaire n 19, Paris, éditions du CTNERHI, 2005.

Villey, Pierre (1914), Le monde des aveugles. Essai de psychologie, Paris, Librairie José Corti/G.I.A.A., 1984, $396 \mathrm{p}$.

Villey, Pierre (1922), La Pédagogie des aveugles, Paris, Librairie Félix Alcan/G.I.A.A., nouvelle édition, s.d., 307 p.

Villey, Pierre (1932), Maurice de La Sizeranne (1857-1924). Aveugle, bienfaiteur des aveugles, Paris, Plon, 126 p.

Weygand, Zina (2003), Vivre sans voir. Les aveugles dans la société française du Moyen Age au siècle de Lovis Braille, Grâne, Créaphis, 372 p. (Préface d'Alain Corbin).

Weygand, Zina (2003) «Les pauvres aveugles dans la société française (début du XIXe siècle) I), dans Gueslin A. et Stiker H.-J. (dir.), Handicaps, pauvreté et exclusion dans la France du XIXe siècle, Paris, Les éditions de l'Atelier, pp. 53-72.

Weygand, Zina (2001) « Un clavier pour les aveugles ou le Destin d'un inventeur : Pierre François Victor 


\section{Zina Weygand}

Foucault (1797-1871) I, VOIR Barré n²3, décembre 2001, pp. 30-41.

\section{Sources écrites}

Aussel, Constant (1901), "Le phonographe et la machine à écrire ॥, Le Valentin Haüy, 1901/8, 19e année, pp. 88-90.

Aussel, Constant (1903), « La dactylo-téléphonographie et les aveugles ॥, Le Valentin Haüy, 1903/8, 21e année, pp. 73-77.

«Blind Telephone Operators », Family Herald Weekly Star. Practical Agriculturist and Popular Magazine, Montréal, Canada, Wednesday, November 27, 1907, p. 1.

Fowler, E.H. (1892), " Type-writing as a vocation for the blind ", The Mentor, published monthly by the Alumni Association for the Perkins Institution for the Blind, Boston, Volume II, 1892/4, pp. 142-143.

Guadet, J. (1859), « De l'accord de pianos par les aveugles », L'Instituteur des aveugles, Journal mensuel, tome IV, 1859/2 et 3, pp. 34-51.

Haüy, Valentin (1786), Essai sur l'éducation des aveugles, avec une préface de Pierre Henri, Paris, Editions des Archives Contemporaines, 1985, 126 p., Annexes, (collection Bibliothèque du Cnam).

Henri, Pierre (1928) «L'emploi des aveugles comme téléphonistes ॥, Extrait de la Revue industrielle d'Août 1928. Copie dactylographiée, Association Valentin Haüy pour le Bien des Aveugles, 10 p.

La Brière, H. (de) (1899), "La dactylographie », Le Valentin Haüy, 1899/9, 17e année, pp. 75-76.

La Sizeranne, Maurice (de) (1883), «Aux amis des aveugles ॥, Le Valentin Haüy, 1883/1, 1ère année, pp. 1-5.

La Sizeranne, Maurice (de) (1890), Dix ans d'études et de propagande en faveur des aveugles, Tournon, Imprimerie de J. Parnin, $418 \mathrm{p}$.

La Sizeranne, Maurice (de) (1910), La Question des aveugles en 1910. Notes et documents, Caen, Imprimerie Adeline G. Poisson et Cie, 170 p.

L'emploi des Aveugles comme Téléphonistes. Notice de propagande, Association Valentin Haüy, 2 p., s.d.

"Les aveugles téléphonistes ", Le Valentin Haüy, $1917 / 2$, p. 12

Lieutenant Renaux (1921), « Professions nouvellement accessibles aux Aveugles. Les téléphonistes », Le Valentin Haüy, 1921/3, pp. 45-47. Suivi de «Attestations en faveur des téléphonistes aveugles ॥, pp. 47-48.

Loury, Eugène (1930), « Les aveugles peuvent faire de bons téléphonistes $»$, La Lumière, numéro du 6 septembre 1930.

Nichols, Homer W. (1935), « Telephone switchboard project for the blind $"$, in Proceedings of the Sixteenth biennial Convention of the American Association of Workers for the Blind, Louisville, Kentucky, June 24 to 28, 1935, pp. 73-78.

Nouvelles Carrières pour Aveugles, Extraits de la Revue mensuelle La Sténo-Mécanographie, Janvier à Mai 1914, 4 p.

OEuvre de placement et de secours en faveur des élèves sortis de l'Institution [œuvre créée le 6 décembre 1849], Institut National des Aveugles. Feuillet de présentation, Paris, 6 décembre 1849, Imprimerie de W. Remquet, $1 \mathrm{p}$.

OEuvre de placement et de secours en faveur des élèves sortis de l'Institution, Institut National des Aveugles. Liste des souscripteurs, 31 décembre 1850, Imprimerie de W. Remquet, $2 \mathrm{p}$.

OEuvre de placement et de secours en faveur des élèves sortis de l'Institution Impériale des Jeunes aveugles, «Maison des ouvriers aveugles de Versailles, créée sous les auspices de l'Institution de Paris». Plaquette de présentation, Imprimerie de W. Remquet, s.d., 3 p.

Société de placement et de secours. Présentation de la Société, suivie de "Société de placement et de secours en faveur des élèves sortis de l'Institution impériale des jeunes aveugles. Première assemblée générale annuelle (16 juin 1856) » Paris, Imprimerie de Mme Ve Dondey-Dupré, $37 \mathrm{p}$.

«Société de placement et de secours en faveur des élèves sortis de l'Institution nationale des jeunes aveugles II, Le Valentin Haüy, 1885/4, 3e année, pp. 25-29.

Société de patronage et de secours pour les aveugles en France [créée en 1841]. Plaquette de présentation, s.d., 4 p.

"Société de Patronage et de Secours pour les aveugles ». Première réunion générale, sous la présidence de M. le comte Portalis, le 18 mai 1843, Annales de l'éducation des sourds-muets et des aveugles. Revue des Institutions qui leur sont consacrées en France et à l'étranger, publiée par M. Edouard Morel, 1 ère année, $1^{\text {er }}$ volume, 1844, pp. 60-61.

"Société de patronage et de secours pour les aveugles ॥. Deuxième séance générale sous la présidence de M. le comte Portalis, 29 janvier 1846, Annales de l'éducation des sourds-muets et des aveugles, 3e année, 3e volume, 1846, pp. 131-143.

"Société de Patronage et de Secours pour les Aveugles Travailleurs " [c'est la même société qui a changé de nom]. Troisième séance générale, sous la présidence de M. Portalis, 5 juillet 1849, Annales de l'éducation des sourds-muets et des aveugles, $6^{\circ}$ année, $6^{e}$ volume, pp. 161-189. 


\section{Les aveugles au travail}

Société de Patronage et de Secours pour les Aveugles Travailleurs en France. Quatrième séance générale, sous la présidence de M. Portalis, 27 juillet 1850 , Paris, Imprimé par E. Thunot et Cie, 1850, 41 p.

La Société d'assistance pour les aveugles (Reconnue d'utilité publique par décret du 4 mars 1886). Son histoire (1879-1915), s.d., $323 \mathrm{p}$.

"Société nationale d'assistance pour les aveugles travailleurs \#. Siège social à l'Hospice National des Quinze-Vingts, 28, rue de Charenton, Paris, Le Valentin Haüy, 1883/3, pp. 19-21 et 1883/4, pp. 25-29 [C'est la même société que précédemment, fondée en 1880 par Alphonse Péphau, directeur des Quinze-Vingts.]

Vives, Juan-Luis (1525), De subventione pauperum. De l'assistance aux pauvres, traduit du latin par le professeur Aznar-Casanova et Léopold Caby, Bruxelles, éditions Valéro et fils, 1943, 290 p. (collection "Vives»)

\section{Entretiens}

Le 24 janvier 2008, Mme Joëlle Lecoq, responsable de formations au Centre de Formation et de Rééducation Professionnelle de l'Association Valentin Haüy (Standardistes: Agents d'Accueil et de Communication ; Télébilletistes). Présidente de l'Association des Standardistes et Agents Techniques Aveugles de France.

4 février 2008, M. Roger Toupet, standardiste retraité. Vice-président fondateur de l'Amicale des Standardistes Aveugles de France en 1960, devenue " Association des Standardistes et Agents Techniques Aveugles de France " pour regrouper tous les emplois de bureau.

10 avril 2008, M. Philippe Chazal, Directeur du Centre de Formation et de Rééducation Professionnelle de l'Association Valentin Haüy. 